\title{
PI3K/Akt/mTOR signalling pathway activation in patients with ER-positive, metachronous, contralateral breast cancer treated with hormone therapy
}

\author{
HIROFUMI KANAIZUMI, CHIHIRO HIGASHI, YUMIKO TANAKA, MIKA HAMADA, WATARU SHINZAKI, \\ TATSUYA AZUMI, YUKIHIKO HASHIMOTO, HIROKI INUI, TOSHIYA HOUJOU and YOSHIFUMI KOMOIKE
}

Division of Breast and Endocrine Surgery, Department of Surgery, Kindai University Faculty of Medicine, Osakasayama, Osaka 589-8511, Japan

Received May 23, 2018; Accepted October 31, 2018

DOI: $10.3892 / 01.2018 .9759$

\begin{abstract}
Oestrogen receptor (ER)-positive, metachronous, contralateral breast cancer (MCBC) sometimes develops during or soon after completion of hormone therapy (HT), but it is uncertain whether it is HT-resistant. We examined the association between ER-positive second cancer and activation of the phosphoinositide 3-kinase (PI3K)/Akt/mammalian target of rapamycin (mTOR) and mitogen-activated protein kinase (MAPK) pathways, which are associated with HT resistance. We examined the treatment-free interval (time after completion of HT for initial cancer) in 41 patients with ER-positive MCBC with a history of adjuvant HT for initial cancer (HT group), and initial-to-second period duration (time after operation of initial cancer to onset of second cancer) in 17 patients with ER-positive MCBC in whom adjuvant HT was not applied to the initial tumour (control group or no HT group). Phosphorylated S6 (pS6) and phosphorylated MAPK (pMAPK) were used as indicators of PI3K/Akt/mTOR and MAPK pathway activity, respectively. Tumours were classified as showing negative, positive or strongly positive staining, and the correlation between staining and treatment-free interval or initial-to-second period duration was evaluated using the Spearman's rank correlation coefficient $(\rho)$. Treatment-free
\end{abstract}

Correspondence to: Dr Hirofumi Kanaizumi, Division of Breast and Endocrine Surgery, Department of Surgery, Kindai University Faculty of Medicine, 377-2 Onohigashi, Osakasayama, Osaka 589-8511, Japan

E-mail: kanaizumi@med.kindai.ac.jp

Abbreviations: $\mathrm{MCBC}$, metachronous, contralateral breast cancer; ER, oestrogen receptor; PI3K, phosphoinositide 3-kinase; mTOR, mammalian target of rapamycin; MAPK, mitogen-activated protein kinase; pS6, phospho-S6 ribosomal protein; HT, hormone therapy; S6K, S6 kinase; PgR, progesterone receptor; HER2, human epidermal growth factor receptor 2; TAM, tamoxifen; AI, aromatase inhibitor

Key words: MCBC, S6, MAPK, mTOR, HT interval and pS6 staining showed a negative correlation ( $\rho=-0.5355 ; P=0.0003$ ) in the HT group. There was no correlation between initial-to-second period duration and pS6 staining in the no HT group $(\rho=-0.0814 ; P=0.756)$. There was no correlation between pMAPK signalling and the treatment-free interval in the HT group ( $\rho=-0.1560 ; \mathrm{P}=0.330)$ or the initial-to-second period duration in the no HT group ( $\rho=-0.0116 ; P=0.965)$. Development of a second ER-positive cancer during or soon after completion of HT for the initial cancer may be associated with activation of the PI3K/Akt/mTOR pathway. Care should be taken during follow-up and when selecting adjuvant therapy for second cancer.

\section{Introduction}

Women with a history of breast cancer are estimated to have an annual probability of developing metachronous, contralateral breast cancer (MCBC) of $0.5-0.75 \%$, and according to the Surveillance, Epidemiology, and End Results data, the probabilities of developing MCBC within 10 and 20 years are 6 and $12 \%$, respectively (1). As advances in drug therapy have resulted in breast cancer patients surviving longer, the number of women at risk of developing MCBC has increased (2), and therefore, more attention must be given to this disease.

$\mathrm{MCBC}$ is known to confer a high risk of distant recurrence and poor prognosis in cases with a short interval between the initial and second cancers. One reason is that if the second cancer is not a primary tumour, it may be a metastasis from the initial tumour. Previous studies comparing gene mutations in initial and second cancers reported that in $6-12 \%$ of cases, the two tumours present with similar genetic mutations $(3,4)$. Another reason is that the influence of long-term adjuvant therapy for the initial cancer may affect the second cancer subtype, leading to a more aggressive tumour or acquired treatment resistance.

In the case of $\mathrm{MCBC}$, the second contralateral breast cancer, unlike the initial cancer, has a history of being exposed to drug treatment and is thus affected by this history. There have been reports that the risk of developing a second cancer is markedly affected by whether postoperative adjuvant hormone therapy (HT) is administered for the initial cancer (5). This suggests 
that the development of a second cancer may be prevented by HT. However, an oestrogen-receptor (ER)-positive second cancer sometimes develops during or soon after completion of postoperative adjuvant HT for the initial cancer. It can therefore be suggested that the growth of this second cancer occurs while under the influence of the HT being administered; thus, despite the ER-positive nature of the second cancer, standard HT may not be effective.

The phosphoinositide 3-kinase (PI3K)/Akt/mammalian target of rapamycin (mTOR) pathway is a cell growth pathway mediated by oestrogen signalling, and abnormal activation of this pathway has been shown to be associated with resistance to HT in ER-positive breast cancer (6). In addition, the mitogen-activated protein kinase (MAPK) pathway is involved in non-oestrogen-dependent cell growth, differentiation, survival, and infiltration, and is an important pathway in relation to resistance to $\mathrm{HT}(7,8)$.

The objective of the present study was to study HTresistance of second cancers that arose during or after completion of adjuvant HT for the initial cancer, by evaluating the relationship between development of ER-positive second cancers and the activation of the PI3K/Akt/mTOR and MAPK pathways, using immunohistochemistry. The treatment-free interval, which is the time between completion of adjuvant HT for the initial cancer and development of the second cancer, was also included in the analysis.

\section{Materials and methods}

Tumour specimens. Eighty-four patients underwent surgery for MCBC as a second cancer at the Kindai University Faculty of Medicine between January 2000 and June 2017. MCBC was defined as contralateral breast cancer diagnosed $>6$ months after surgery for the initial cancer; the second cancer was not evaluated to determine whether it was primary or metastatic in nature. Three patients were excluded from the analysis since surgery for the initial cancer was performed at a different hospital and the details and duration of the postoperative adjuvant therapy were unknown, and two were excluded owing to the detection of local or distant metastases before development of the second cancer. Of the 79 remaining patients, 58 had an ER-positive second cancer. Postoperative adjuvant HT was administered for the initial cancer in 41 patients, who formed the subject group in the present study (HT group). No adjuvant HT was administered for the initial cancer in 17 patients, who formed the control group (no HT group). The present study was retrospective in nature. Initially, the study subjects were included from 2006 to 2015. This research plan was approved by the Kindai University Faculty of Medicines Ethics Committee on 26th May 2017. However, since the number of cases was lesser than we expected, we revised the study period, and subjects were included from January 2000 to June 2017. This revised study period was approved by the Ethics Committee on 20th February 2018.

Immunohistochemistry. When preparing tissue specimens, in order to ensure the stability of phosphorylated proteins in the surgical samples, excised tissue samples were fixed for 24 to $48 \mathrm{~h}$ with $10 \%$ buffered formalin immediately after collection, after which they were embedded in paraffin and sectioned to prepare 4- $\mu \mathrm{m}$-thick slices. As an index of PI3K/Akt/mTOR pathway activation, immunohistochemistry was carried out for phospho-S6 ribosomal protein (pS6) expression $(9,10)$; pS6 is a protein which is phosphorylated by $\mathrm{S} 6$ kinase (S6K; a downstream molecule in the PI3K/Akt/mTOR signalling pathway), and is an important factor in regulating translation $(11,12)$. In addition, immunohistochemistry was carried out for pMAPK expression as an index of MAPK pathway activation $(9,13)$. The primary antibodies used were D57.2.2E [phospho-S6 ribosomal protein (Ser235/236), rabbit monoclonal antibody; Cell Signaling Technology, Inc., Danvers, MA, USA], and D13.14.4E [phospho-p44/42 MAPK (Erk1/2) (Thr202/Tyr204), XP ${ }^{\circledR}$ rabbit monoclonal antibody; Cell Signaling Technology, Inc.]. After deparaffinisation of the sections, antigen retrieval was carried out by heating the slides to $98^{\circ} \mathrm{C}$ for 40 min in citric acid ( $\mathrm{pH}$ 6.0). Endogenous peroxidase activity was blocked by treatment with $0.3 \%$ hydrogen peroxide, and non-specific binding was blocked by treatment for $10 \mathrm{~min}$ with $5 \%$ normal goat serum. The primary antibodies were each diluted 400 -fold and added to the slides, which were incubated overnight at $4^{\circ} \mathrm{C}$. After removal of the primary antibodies, Histofine Simple Stain MAX-PO (Multi; Nichirei Corporation, Tokyo, Japan) was added to the slides drop-wise, followed by incubation for $30 \mathrm{~min}$. Finally, we used the Liquid DAB+, two-component system (Dako Corporation, Santa Clara, CA, USA) for detection. We added 1 drop of the DAB Chromogen per ml of substrate buffer, and staining was carried out for $1 \mathrm{~min}$. Counterstaining was also carried out according to the manufacturer's instructions.

ER and progesterone-receptor (PgR) expressions were analysed according to the new American Society of Clinical Oncology guidelines; specimens with at least $1 \%$ of stained cells were defined as positive. Specimens were defined as positive for human epidermal growth factor receptor 2 (HER2) expression if they were scored as $3+$ by immunohistochemistry or as $2+$ by immunohistochemistry and were positive by fluorescent in situ hybridisation. Whether Ki67 was high or low was based on a $14 \%$ positivity cut-off value (14). The subtype of Luminal B was assumed to have high Ki67 or a negative PgR status. Analysis of pS6 and pMAPK staining used the H-score, as reported previously (15-17). Briefly, 10 high-powered fields (magnification, x200) were observed using light microscopy, and the mean proportion of each field occupied by positive cells was calculated and accorded a numeric value as follows: 0: $0-5 \%$; 1: $6-25 \% ; 2: 26-50 \% ; 3: 51-75 \%$; or $4: 76-100 \%$. In addition, the staining intensity was accorded a numeric value as follows: 0 : negative; 1 : weak; 2 : moderate; or 3 : strong. The numeric value for the proportion of positive cells was then multiplied by that for staining intensity to obtain the H-score; the scores were classified as follows: 0-1: negative; 2-4: positive; and 5-12: strongly positive (Fig. 1). The positive controls used for staining intensity were IHC controls \#8101 for pS6 and \#8103 for pMAPK (SignalSlide ${ }^{\circledR}$; Cell Signaling Technology, Inc.). Immunohistochemical analysis was performed independently by two doctors. Cases of H-score 1-4, for which a consensus can be difficult, were additionally analysed by a pathologist. The slides were numerically coded to ensure that the person performing the analysis was blinded to the patient's clinical background.

Statistical analysis. The relationship between administration of adjuvant HT for the primary cancer (HT group vs. no HT 

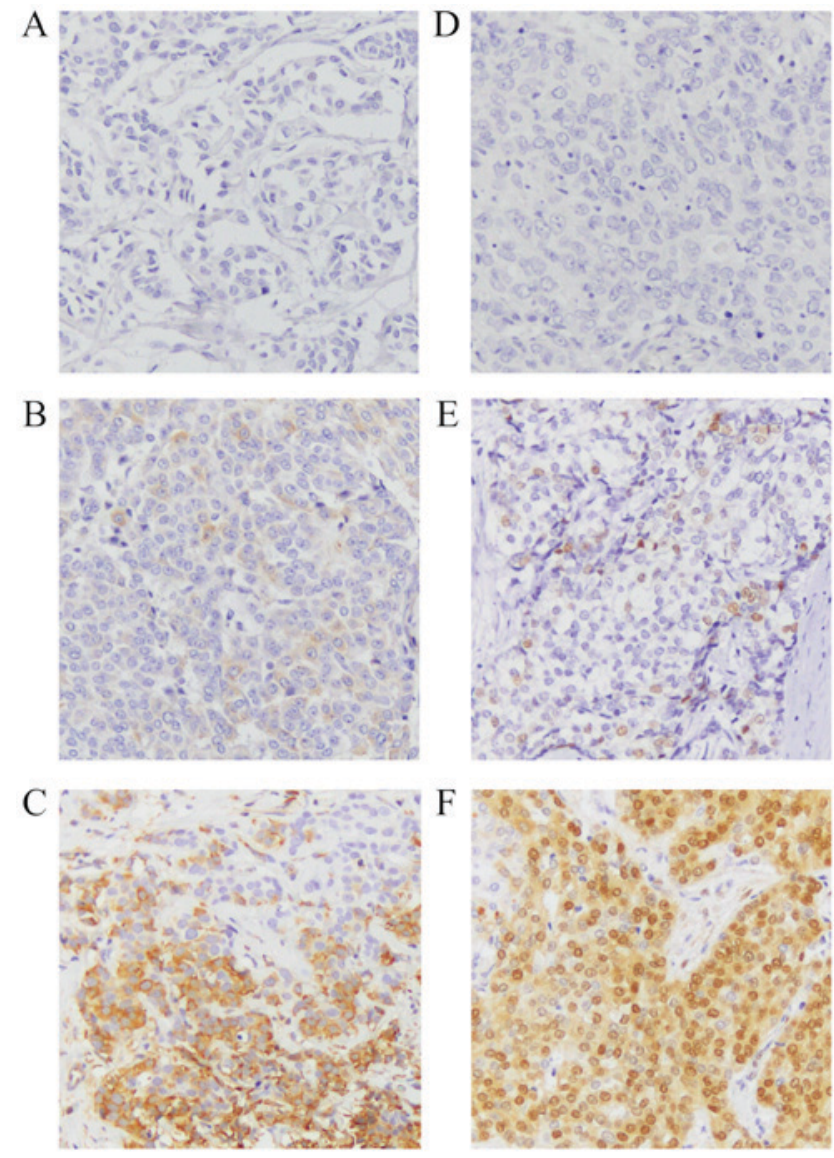

Figure 1.H-score for phospho-S6 ribosomal protein (pS6) and phosphomitogen-activated protein kinase (pMAPK) (magnification, x200). Representative immunostaining images of samples with (A) negative (H-score 0-1), (B) positive (H-score 2-4), and (C) strongly positive (H-score 5-12) staining for pS6, (D) negative (H-score 0-1), (E) positive (H-score 2-4), and (F) strongly positive (H-score 5-12) staining for pMAPK.

group) and clinicopathological background factors was tested using the Fisher's exact test. The clinicopathological characteristics included age at diagnosis of the initial cancer, history of chemotherapy or trastuzumab therapy for the initial cancer, interval between the initial cancer and onset of the second cancer, and characteristics of the initial and second cancer such as stage, status of ER, PgR, HER2, and Ki67 expression, and subtype. A $\mathrm{P}<0.05$ defined a statistically significant difference. The correlation between treatment-free interval or initial-to-second period and the immunohistochemistry results was determined using the Spearman's rank correlation coefficient. The treatment-free interval of patients who developed a second cancer during hormonal therapy was defined as 0 months. The initial-to-second period of the control group was defined as the period from surgery for the initial cancer to onset of the second cancer. The software used in the analysis was JMP ${ }^{\circledR} 13$ (SAS Institute, Inc., Cary, NC, USA).

\section{Results}

We compared the clinicopathological background of initial cancer between patients in the HT group $(n=41)$ and those in the no HT group ( $n=17$ ) (Table I). There was no difference in age at onset, T-stage, and lymph node status of the initial cancer. ER status was positive in all cases in the HT group.
Table I. Association of clinicopathological background with initial cancer between patients in the HT group and those in the no HT group.

\begin{tabular}{lccc}
\hline $\begin{array}{l}\text { Clincopathological } \\
\text { criteria }\end{array}$ & $\begin{array}{c}\text { HT group } \\
(\mathrm{n}=41)\end{array}$ & $\begin{array}{c}\text { No HT } \\
\text { group }(\mathrm{n}=17)\end{array}$ & P-value \\
\hline
\end{tabular}

Age at diagnosis, years

$\begin{array}{llll}<50 & 25 & 8 & 0.390 \\ \geq 50 & 16 & 9 & \end{array}$

T-stage

T1

T2-4

17

4

Lymph node status

Absence

Presence

34

15

1.000

ER status

Positive

Negative

7

2

41

6

0.001

N/A

2

PgR status

Positive

Negative

39

6

9

N/A

2

HER2 status

Positive

Negative

N/A

$\begin{array}{rr}6 & 4 \\ 33 & 11 \\ 2 & 2 \\ 35 & 14 \\ 4 & 1 \\ 2 & 2\end{array}$

0.438

Ki67 status (\%)

$$
<14
$$

$\geq 14$

N/A

Subtype

Luminal A

Luminal B

HER2 enriched

Basal like

N/A

HT

TAM ( 2 years $)$
TAM (5-10 years)

Switch from

TAM to AI

AI (5 years)

Chemotherapy

Yes

No

20

7

0.570

Trastuzumab

Yes

$\begin{array}{rr}2 & 2 \\ 39 & 15\end{array}$

0.573

No

HT group, patients who received hormone therapy for the initial cancer; no HT group, patients who did not receive hormone therapy for the initial cancer; ER, oestrogen receptor; PgR, progesterone-receptor; HER2, human epidermal growth factor receptor 2; TAM, tamoxifen; $\mathrm{AI}$, aromatase inhibitor; N/A, not available. 
Table II. Association of clinicopathological background with second cancer between patients in the HT group and those in the no HT group.

\begin{tabular}{|c|c|c|c|}
\hline $\begin{array}{l}\text { Clincopathological } \\
\text { criteria }\end{array}$ & $\begin{array}{l}\text { HT group } \\
\quad(n=41)\end{array}$ & $\begin{array}{c}\text { No HT } \\
\text { group }(n=7)\end{array}$ & P-value \\
\hline \multicolumn{4}{|c|}{ Age at diagnosis, years } \\
\hline$<50$ & 12 & 7 & 0.540 \\
\hline$\geq 50$ & 29 & 10 & \\
\hline \multicolumn{4}{|l|}{ T-stage } \\
\hline $\mathrm{T} 1$ & 34 & 17 & 0.093 \\
\hline $\mathrm{T} 2-4$ & 7 & 0 & \\
\hline \multicolumn{4}{|l|}{ Lymph node } \\
\hline Absence & 30 & 14 & 0.523 \\
\hline Presence & 11 & 3 & \\
\hline \multicolumn{4}{|l|}{ PgR status } \\
\hline Positive & 36 & 17 & 0.307 \\
\hline Negative & 5 & 0 & \\
\hline \multicolumn{4}{|l|}{ HER2 status } \\
\hline Positive & 6 & 3 & 1.000 \\
\hline Negative & 35 & 14 & \\
\hline \multicolumn{4}{|l|}{ Ki67 status } \\
\hline$<14 \%$ & 25 & 14 & 0.137 \\
\hline$\geq 14 \%$ & 16 & 3 & \\
\hline \multicolumn{4}{|l|}{ Subtype } \\
\hline Luminal A & 20 & 13 & - \\
\hline Luminal B & 15 & 1 & \\
\hline HER2 enriched & 6 & 3 & \\
\hline
\end{tabular}

HT group, patients who received hormone therapy for the initial cancer; no HT group, patients who did not receive hormone therapy for the initial cancer; PgR, progesterone-receptor; HER2, human epidermal growth factor receptor 2; Ki67, proliferation marker protein Ki67.

Though ER status of six patients in the no HT group was positive, they did not receive HT. Of the six patients who did not receive HT, two patients reported adverse events immediately after the administration of the first dose of HT, so did not receive the full therapy. Four other patients presented with microinvasion ( $\leqq 5 \mathrm{~mm}$ ), and opted out of drug treatment for this reason. PgR positivity was significantly higher in the HT group. There was no difference in HER2 status and Ki67 status between the two groups. Cancer subtype data for both the groups are shown in Table I. Immunostaining was not performed in two cases of first cancer in HT and no HT groups due to insufficient amount of tissue. Regarding HT for the initial cancer, most patients were treated with tamoxifen (TAM); while 19 patients received treatment for 2 years, 16 patients received treatment for 5-10 years. Most of the patients who received treatment only for 2 years were treated according to the conventional standard of treatment. One patient was switched from treatment with TAM (1 year) to that with an aromatase inhibitor (AI) (4 years). Five patients were treated with AI (5 years). There was also no difference
Table III. Association of immunostaining with initial and second cancer in the HT group.

\begin{tabular}{|c|c|c|c|}
\hline $\begin{array}{l}\text { Clincopathological } \\
\text { criteria }\end{array}$ & $\begin{array}{l}\text { Initial } \\
\text { cancer }\end{array}$ & $\begin{array}{l}\text { Second } \\
\text { cancer }\end{array}$ & $\mathrm{P}$-value \\
\hline \multicolumn{4}{|l|}{ PgR status } \\
\hline Positive & 39 & 36 & \multirow[t]{2}{*}{0.432} \\
\hline Negative & 2 & 5 & \\
\hline \multicolumn{4}{|l|}{ HER2 status } \\
\hline Positive & 6 & 6 & \multirow[t]{2}{*}{1.000} \\
\hline Negative & 35 & 35 & \\
\hline \multicolumn{4}{|l|}{ Ki67 status } \\
\hline$<14 \%$ & 35 & 25 & \multirow[t]{3}{*}{0.004} \\
\hline$\geq 14 \%$ & 4 & 16 & \\
\hline $\mathrm{N} / \mathrm{A}$ & 2 & 0 & \\
\hline \multicolumn{4}{|l|}{ Subtype } \\
\hline Luminal A & 28 & 20 & \multirow[t]{4}{*}{-} \\
\hline Luminal B & 5 & 15 & \\
\hline HER2 enriched & 6 & 6 & \\
\hline $\mathrm{N} / \mathrm{A}$ & 2 & 0 & \\
\hline \multicolumn{4}{|l|}{ pS6 status } \\
\hline Positive & 6 & 9 & \multirow[t]{3}{*}{0.570} \\
\hline Negative & 33 & 32 & \\
\hline $\mathrm{N} / \mathrm{A}$ & 2 & & \\
\hline \multicolumn{4}{|l|}{ pMAPK status } \\
\hline Positive & 11 & 10 & \multirow[t]{3}{*}{0.801} \\
\hline Negative & 28 & 31 & \\
\hline N/A & 2 & & \\
\hline
\end{tabular}

HT group, patients who received hormone therapy for the initial cancer; PgR, progesterone-receptor; HER2, human epidermal growth factor receptor 2; pS6, phospho-S6 ribosomal protein; pMAPK, phospho-mitogen-activated protein kinase; Ki67, proliferation marker protein Ki67; N/A, not available.

in the frequency of adjuvant chemotherapy or trastuzumab treatment for the initial cancer between the two groups. We also compared the clinicopathological background of second cancers (Table II). There was no difference in age at second cancer onset between the two groups. Although all patients in the no HT group had T1 stage second cancer, there was no significant difference in stage between the two groups. In addition, there was no difference in the pathological background (ER, PgR, HER2, Ki67 status) regarding the subtype of the second cancer or the presence or absence of lymph node metastasis. The median interval between the initial cancer and the second cancer onset was 104 months (9-261 months) for the HT group and 84 months (6-234 months) for the no HT group. In the HT group, the median treatment-free interval was 48 months, and the range was 0-241 months. There were seven patients diagnosed with a second tumour during postoperative adjuvant HT for the initial tumour.

We compared the immunostaining results of initial and second cancers in the HT group (Table III). Although there was no difference in the frequency of PgR and HER2 positivity, the 

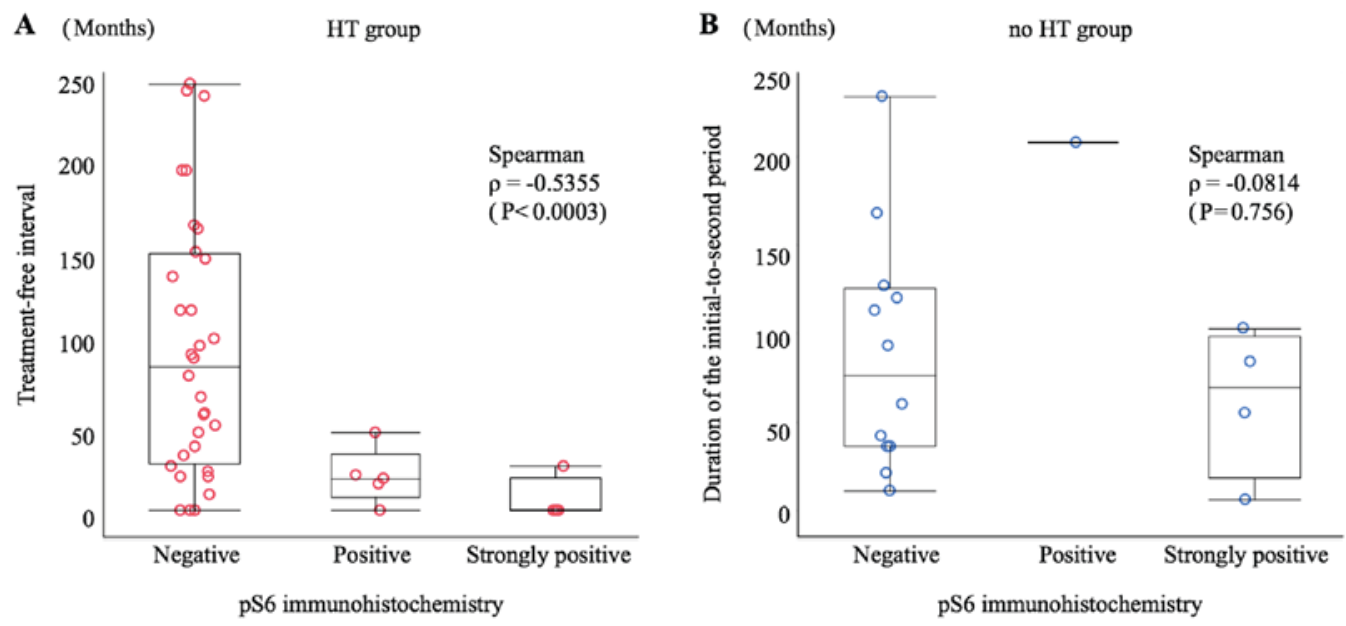

Figure 2. Scatter graph showing the relationship between treatment-free interval or initial-to-second period duration and phospho-S6 ribosomal protein (pS6) immunostaining. (A) HT group: Patients who received HT after diagnosis of the initial tumour; the treatment-free interval of pS6 immunohistochemistry-negative patients was $91.5 \pm 73.6$ months, that of pS6-positive patients was $19.4 \pm 15.8$ months, and that of pS6-strongly positive patients was $6.3 \pm 12.5$ months. There was a rank correlation between the treatment-free interval and pS6 immunostaining $(\mathrm{P}<0.0003)$ according to Spearman correlation analysis $(\rho=-0.5355)$. (B) No HT group: Patients who did not receive HT after diagnosis of the initial tumour; the initial-to-second period duration of pS6 immunohistochemistry-negative patients was $88.4 \pm 67.2$ months, that of pS6-positive patients was $208 \pm 0$ months, and that of pS6-strongly positive patients was $62.0 \pm 42.2$ months. There was no rank correlation between the initial-to-second period duration and pS6 immunostaining $(\mathrm{P}=0.756)$ according to Spearman correlation analysis $(\rho=-0.0814)$. HT, hormone therapy.
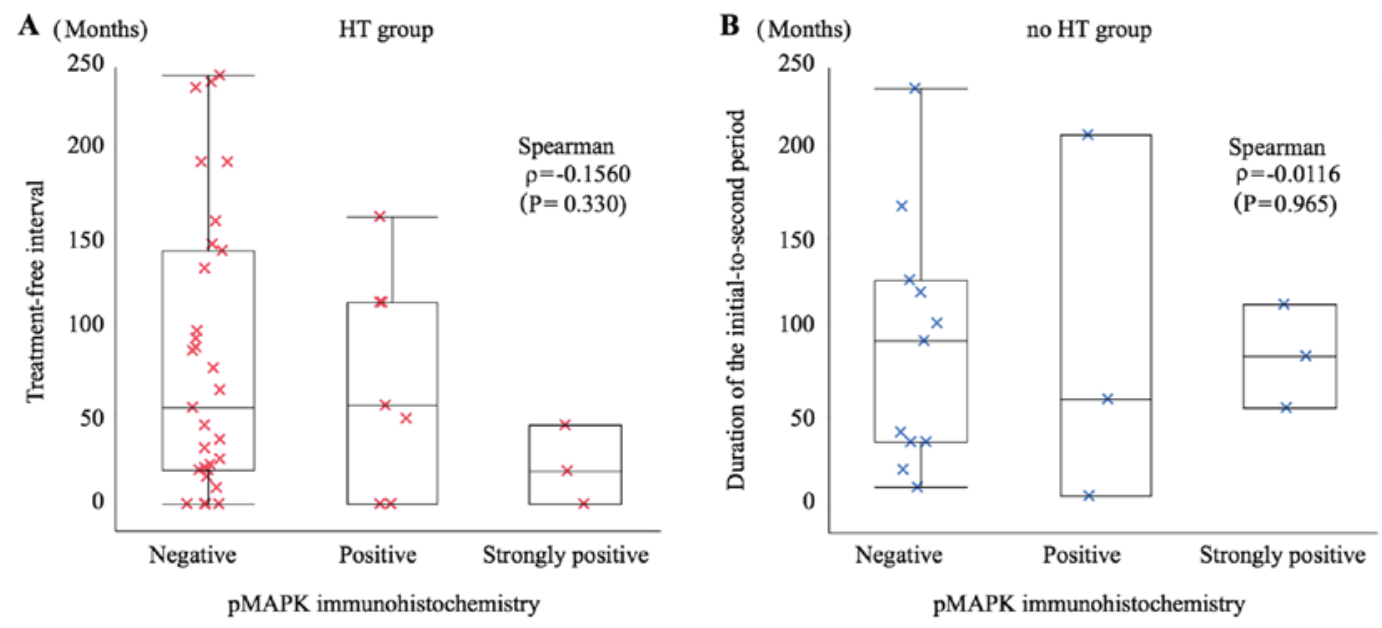

Figure 3. Scatter graph showing the relationship between treatment-free interval or initial-to-second period duration and phospho-mitogen-activated protein kinase (pMAPK) immunostaining. (A) HT group: Patients who received HT after diagnosis of the initial tumour; the treatment-free interval of pMAPK immunohistochemistry-negative patients was $80.6 \pm 77.2$ months, that of pMAPK-positive patients was $70.0 \pm 61.2$ months, and that of pMAPK-strongly positive patients was $20.7 \pm 22.1$ months. There was no rank correlation between the treatment-free interval and pMAPK immunostaining $(\mathrm{P}=0.330)$ according to Spearman correlation analysis ( $\rho=-0.1560$ ). (B) No HT group: Patients who did not receive HT after diagnosis of the initial tumour; the initial-to-second period duration of pMAPK immunohistochemistry-negative patients was $90.1 \pm 69.6$ months, that of pMAPK-positive patients was $91.3 \pm 104.6$ months, and that of pMAPK-strongly positive was $84.0 \pm 29.0$ months. There was no rank correlation between the initial-to-second period duration and pMAPK immunostaining $(\mathrm{P}=0.965)$ according to Spearman correlation analysis $(\rho=-0.0116)$. HT, hormone therapy.

incidence of a Ki67 positive status was significantly higher in the second cancers. As a result, the incidence of the luminal B subtype was large in the second cancers. The pS6 positivity of the second cancers increased slightly compared to that of the initial cancers, though the difference was not significant. There was no difference in MAPK positivity between the initial and second cancers as well.

In order to ascertain the relationship between treatment-free interval or initial-to-second period duration and immunohistochemistry results, a scatter graph was prepared. The Spearman's rank correlation coefficient $(\rho)$ for pS6 immunohistochemistry and treatment-free interval in the HT group was $\rho=-0.5355(\mathrm{P}=0.0003)$, indicating a negative correlation, i.e. a tendency towards a high to strong frequency of pS6 positivity with a short treatment-free interval. However, this trend was not observed in the relationship between pS6 immunohistochemistry and initial-to-second period duration in the no HT group ( $\rho=-0.0814 ; \mathrm{P}=0.756)$ (Fig. 2). There was no correlation between pMAPK signalling and the treatment-free interval in the HT group $(\rho=-0.1560 ; \mathrm{P}=0.330)$ or the initial-to-second period duration in the no HT group ( $\rho=-0.0116 ; \mathrm{P}=0.965$ ) (Fig. 3). 


\section{Discussion}

The results of the present study show that in ER-positive MCBCs in patients with a history of HT, the frequency of pS6 positivity increases with decreasing treatment-free interval. This suggests that, despite the second cancer being ER-positive, the PI3K/Akt/mTOR pathway is activated in these tumours owing to the effect of HT for the initial cancer, resulting in resistance to HT.

According to a previous study, pS6 expression was elevated in a murine model of ER- and PgR-positive breast cancer treated with preoperative adjuvant HT, and the expression level was linked to the efficacy of mTOR inhibition (18). In addition, there have recently been reports that the effects of HT include development of resistance to the therapy. According to studies of metastatic foci of breast cancer, patients who are administered adjuvant HT have metastatic foci that are significantly more positive for Akt, mTOR, and phosphorylated S6K1 than do patients not treated with HT, suggesting activation of the PI3K/Akt/mTOR signalling pathway through oestrogen blockade (19). It has also been reported that $\mathrm{S} 6 \mathrm{~K}$-positive patients, in comparison to S6K-negative patients, did not show reduced risk of cancer recurrence, even when administered tamoxifen as adjuvant therapy (20). In our study, there was no increase in the frequency of pS6 positivity due to endocrine treatment history; however, our results showed a significant correlation between the time to diagnosis of a second cancer after completion of HT and the frequency and intensity of pS6 expression. We consider that this correlation may potentially affect the success of treatment regimens and should be used to guide treatment decisions for second cancers with short treatment-free intervals.

No relationship was found between MAPK pathway activation and time to diagnosis of a second cancer after HT. The results of an in vitro study suggested that long-term survival of a cancer cell line cultured under conditions of oestrogen depletion is more markedly dependent on the PI3K/Akt/mTOR pathway than it does on the MAPK pathway $(21,22)$, which is in agreement with our findings.

One limitation to the present study is that since MCBC is a relatively uncommon disease, our cohort was small; thus, we could not make definitive conclusions based on our study results. The trends seen in our findings are significant and interesting, but the correlation coefficients were not strong. Moreover, in this case, since the follow-up period from the onset of the second cancer was short and the number of relapses and deaths was small, we could not determine the prognosis of the cases that were positive for pS6. In addition, the type and duration of endocrine treatment for the first cancer could affect pS6 expression of the second cancer. However, in our case, there were several treatment periods and types, so we could not analyse that.

In conclusion, we found that in patients with ER-positive breast cancer treated with HT, rapid development of an ER-positive second cancer correlated with a higher frequency of pS6 positivity. With this type of second cancer, the likelihood of activating the PI3K/Akt/mTOR pathway is high, and selection of adjuvant therapy regimens and follow-up should be performed accordingly.

\section{Acknowledgements}

The authors would like to thank Professor Takao Sato ( (Department of Pathology, Kindai University Faculty of Medicine) for their help in analysing immunostaining spcimens. The preliminary result of the present study was presented at The 11th European Breast Cancer Conference in March 2018.

\section{Funding}

No funding was received.

\section{Availability of data and materials}

The datasets used and/or analysed during the present study are available from the corresponding author on reasonable request.

\section{Authors' contributions}

YK designed the study. HK analysed the data and wrote the paper. $\mathrm{YK}$ and $\mathrm{HK}$ analysed the immunostained specimens. $\mathrm{CH}$, YT, MH, WS, TA, YH, HI and TH collected the data, critically evaluated the data analysis and analysis of immunostained specimens, and contributed to the construction of important intellectual content. All authors read and approved the final manuscript.

\section{Ethics approval and consent to participate}

The present study has been approved by the Research Ethics Committee of the Kindai University (Osakasayama, Japan). We explained to all patients who underwent an operation that we may use the resected tissues for research purposes. In the present study, all patients agreed to this. We also explained the present study to the outpatients and obtained their consent for participation. We provided a means to opt out to patients who discontinued their visits and to the families of deceased patients. An explanatory document (research document) on the research approved by the Ethics Committee was published on the website (http://www.kindai-geka.jp/). Questions and consultation concerning the present study (subjects such as the implementation plan, analysis results, etc.) from patients and families were accepted at any time on the website, and the opportunity to refuse participation was provided.

\section{Patient consent for publication}

Not applicable.

\section{Competing interests}

The authors declare that they have no competing interests.

\section{References}

1. Gao X, Fisher SG and Emami B: Risk of second initial cancer in the contralateral breast in women treated for early-stage breast cancer: A population-based study. Int J Radiat Oncol Biol Phys 56: 1038-1045, 2003. 
2. Jobsen JJ, van der Palen J, Ong F, Riemersma S and Struikmans H: Bilateral breast cancer, synchronous and metachronous; differences and outcome. Breast Cancer Res Treat 153: 277-283, 2015.

3. Klevebring D, Lindberg J, Rockberg J, Hilliges C, Hall P, Sandberg M and Czene K: Exome sequencing of contralateral breast cancer identifies metastatic disease. Breast Cancer Res Treat 151: 319-324, 2015.

4. Begg CB, Ostrovnaya I, Geyer FC, Papanastasiou AD, Ng CKY, Sakr RA, Bernstein JL, Burke KA, King TA, Piscuoglio S, et al: Contralateral breast cancers: Independent cancers or metastases? Int J Cancer 142: 347-356, 2018.

5. Schaapveld M, Visser O, Louwman WJ, Willemse PH de Vries EG, van der Graaf WT, Otter R, Coebergh JW and van Leeuwen FE: The impact of adjuvant therapy on contralateral breast cancer risk and the prognostic significance of contralateral breast cancer: A population based study in the Netherlands. Breast Cancer Res Treat 110: 189-197, 2008.

6. Miller TW, Balko JM and Arteaga CL: Phosphatidylinositol 3kinase and antiestrogen resistance in breast cancer. J Clin Oncol 29: 4452-4461, 2011.

7. Shou J, Massarweh S, Osborne CK, Wakeling AE, Ali S, Weiss H and Schiff R: Mechanisms of tamoxifen resistance: Increased estrogen receptor-HER2/neu cross-talk in ER/HER2-positive breast cancer. J Natl Cancer Inst 96: 926-935, 2004.

8. Al Saleh S, Sharaf LH and Luqmani YA: Signalling pathways involved in endocrine resistance in breast cancer and associations with epithelial to mesenchymal transition (Review). Int J Oncol 38: 1197-1217, 2011.

9. Yanai A, Inoue N, Yagi T, Nishimukai A, Miyagawa Y, Murase K Imamura M, Enomoto Y, Takatsuka Y, Watanabe T, et al: Activation of mTOR/S6K but not MAPK pathways might be associated with High Ki-67, ER ${ }^{+}$, and HER2- breast cancer. Clin Breast Cancer 15: 197-203, 2015.

10. Sun $\mathrm{CH}$, Chang $\mathrm{YH}$ and Pan CC: Activation of the $\mathrm{PI} 3 \mathrm{~K} / \mathrm{Akt} / \mathrm{mTOR}$ pathway correlates with tumour progression and reduced survival in patients with urothelial carcinoma of the urinary bladder. Histopathology 58: 1054-1063, 2011.

11. Fingar DC, Richardson CJ, Tee AR, Cheatham L, Tsou C and Blenis J: mTOR controls cell cycle progression through its cell growth effectors S6K1 and 4E-BP1/eukaryotic translation initiation factor 4E. Mol Cell Biol 24: 200-216, 2004.

12. Mamane Y,Petroulakis E, LeBacquer O and Sonenberg N: mTOR, translation initiation and cancer. Oncogene 25: 6416-6422, 2006.

13. Bose S, Chandran S, Mirocha JM and Bose N: The Akt pathway in human breast cancer: A tissue-array-based analysis. Mod Pathol 19: 238-245, 2006.
14. Cheang MC, Chia SK, Voduc D, Gao D, Leung S, Snider J, Watson M, Davies S, Bernard PS, Parker JS, et al: Ki67 index, HER2 status, and prognosis of patients with luminal B breast cancer. J Natl Cancer Inst 101: 736-750, 2009.

15. Song CH, Park SY, Eom KY, Kim JH, Kim SW, Kim JS and Kim IA: Potential prognostic value of heat-shock protein 90 in the presence of phosphatidylinositol-3-kinase overexpression or loss of PTEN, in invasive breast cancers. Breast Cancer Res 12: R20, 2010.

16. Brewster AM and Parker PA: Current knowledge on contralateral prophylactic mastectomy among women with sporadic breast cancer. Oncologist 16: 935-941, 2011.

17. Liu Z, Yun R, Yu X, Hu H, Huang G, Tan B and Chen T: Overexpression of Notch3 and pS6 is associated with poor prognosis in human ovarian epithelial cancer. Mediators Inflamm 2016: 5953498, 2016.

18. Polo ML, Riggio M, May M, Rodríguez MJ, Perrone MC, Stallings-Mann M, Kaen D, Frost F, Goetz M, Boughey J, et al: Activation of PI3K/Akt/mTOR signaling in the tumor stroma drives endocrine therapy-dependent breast tumor regression. Oncotarget 6: 22081-22097, 2015.

19. Beelen K, Hoefnagel LD, Opdam M, Wesseling J, Sanders J, Vincent AD, van Diest PJ and Linn SC: PI3K/AKT/mTOR pathway activation in initial and corresponding metastatic breast tumors after adjuvant endocrine therapy. Int J Cancer 135: 1257-1263, 2014

20. Kim EK, Kim HA, Koh JS, Kim MS, Kim KI, Lee JI, Moon NM, Ko E and Noh WC: Phosphorylated S6K1 is a possible marker for endocrine therapy resistance in hormone receptor-positive breast cancer. Breast Cancer Res Treat 126: 93-99, 2011.

21. Ray S and Darbre PD: Crosstalk with insulin and dependence on $\mathrm{PI} 3 \mathrm{~K} / \mathrm{Akt} / \mathrm{mTOR}$ rather than MAPK pathways in upregulation of basal growth following long-term oestrogen deprivation in three human breast cancer cell lines. Horm Mol Biol Clin Investig 5: 53-65, 2011.

22. Martin LA, Farmer I, Johnston SR, Ali S, Marshall C and Dowsett M: Enhanced estrogen receptor (ER) alpha, ERBB2, and MAPK signal transduction pathways operate during the adaptation of MCF-7 cells to long term estrogen deprivation. J Biol Chem 278: 30458-30468, 2003. 